\title{
The predictive value of the preoperative C-reactive protein-albumin ratio for early recurrence and chemotherapy benefit in patients with gastric cancer after radical gastrectomy: using randomized phase III trial data
}

\author{
Bin-bin $X u^{1,2,3}$. Jun Lu ${ }^{1,2,3} \cdot$ Zhi-fang Zheng ${ }^{1,2,3}$. Jian-wei Xie ${ }^{1,2,3}$. Jia-bin Wang ${ }^{1,2,3}$. Jian-xian Lin 1,2,3 \\ Qi-yue Chen ${ }^{1,2,3} \cdot$ Long-long Cao ${ }^{1,2,3} \cdot$ Mi Lin $^{1,2,3} \cdot$ Ru-hong Tu ${ }^{1,2,3} \cdot$ Ze-ning Huang ${ }^{1,2,3} \cdot$ Ju-li Lin ${ }^{1,2,3}$. \\ Chao-hui Zheng ${ }^{1,2,3}$. Chang-ming Huang ${ }^{1,2,3} \cdot$ Ping Li $\mathrm{Li}^{1,2,3}$
}

Received: 18 November 2018 / Accepted: 2 February 2019 / Published online: 9 February 2019

(c) The International Gastric Cancer Association and The Japanese Gastric Cancer Association 2019

\begin{abstract}
Background The definition and predictors of early recurrence (ER) for gastric cancer (GC) patients after radical gastrectomy are unclear.

Methods A minimum- $p$ value approach was used to evaluate the optimal cutoff value of recurrence-free survival to determine ER and late recurrence (LR). Receiver operating characteristic curves were generated for inflammatory indices. Potential risk factors for ER were assessed with a Cox regression model. A decision curve analysis was performed to evaluate the clinical utility.

Results A total of 401 patients recruited in a clinical trial (NCT02327481) from January 2015 to April 2016 were included in this study. The optimal length of recurrence-free survival to distinguish between ER $(n=44)$ and LR $(n=52)$ was 12 months. Factors associated with ER included a preoperative C-reactive protein-albumin ratio (CAR) $\geq 0.131$, stage III and postoperative adjuvant chemotherapy (PAC) $>3$ cycles. The risk model consisting of both the CAR and TNM stage had a higher predictive ability and better clinical utility than TNM stage alone. Further stratification analysis of the stage III patients found that for the patients with a CAR $<0.131$, both PAC with $1-3$ cycles $(p=0.029)$ and $>3$ cycles $(p<0.001)$ could reduce the risk of ER. However, for patients with a CAR $\geq 0.131$, a benefit was observed only if they received PAC $>3$ cycles $(54.2 \%$ vs $16.0 \%, p=0.004)$, rather than $1-3$ cycles $(58.3 \%$ vs $54.2 \%, p=0.824)$.

Conclusions A recurrence-free interval of 12 months was found to be the optimal threshold for differentiating between ER and LR. Preoperative CAR was a promising predictor of ER and PAC response. PAC with 1-3 cycles may not exert a protective effect against ER for stage III GC patients with CAR $\geq 0.131$.
\end{abstract}

Keywords Gastric cancer $\cdot$ Early recurrence $\cdot$ Adjuvant chemotherapy $\cdot$ C-reactive protein-albumin ratio $\cdot$ Post-recurrence survival

Jun Lu and Bin-bin Xu contributed equally to this work and should be considered co-first authors.

Electronic supplementary material The online version of this article (https://doi.org/10.1007/s10120-019-00936-w) contains supplementary material, which is available to authorized users.

Extended author information available on the last page of the article

\section{Introduction}

Gastric cancer (GC) is the fifth most common malignancy in humans and ranks third in tumor-related mortality [1]. Although scholars have worked hard to improve the diagnosis and treatment of gastric cancer, the recurrence rate of patients with gastric cancer is still high - approximately $18-45.5 \%$ [2-4] —and the long-term survival rates are still not optimal $[5,6]$.

Many studies have shown that early recurrence heralds a worse prognosis after radical tumor resection for several 
tumor types, such as pancreatic cancer, lung cancer, colorectal cancer, liver cancer and intrahepatic cholangiocarcinoma [7-11]. Although the term "early recurrence" is often utilized in both the academic and clinical setting, a clear definition is currently lacking, with arbitrary cutoff values varying between 12 and 36 months reported in the literature [12-15]. Therefore, in this study, we used prospective clinical trial data to establish an evidence-based cutoff value to differentiate between early and late recurrence based on the difference in prognosis after recurrence and to identify independent risk factors for early GC recurrence after radical gastrectomy. An evidence-based cutoff value for early recurrence has the potential to aid clinicians in the prognostic stratification of post-gastrectomy patients, and the identified risk factors reported herein could help guide adjuvant treatment decisions.

\section{Materials and methods}

\section{Study population}

Between January 1, 2015, and April 1, 2016, a total of 438 patients admitted to Fujian Medical University Union Hospital were recruited to the study, and 419 patients were ultimately included in the final analysis [16] (ClinicalTrials.gov number NCT02327481). Details on the inclusion, exclusion, quality control, and randomization were previously reported $[16,17]$. The present study is a substudy of the above-mentioned RCT. After excluding 10 patients with neuroendocrine carcinoma, 6 patients with palliative surgery and 2 patients without evidence of GC, the present analysis was restricted to 401 patients for whom curative gastrectomies were performed and for whom postoperative pathology confirmed stage I, II, or III gastric adenocarcinoma (pT1-4aN0-3M0) according to the 7th American Joint Committee on Cancer staging [18]. The study flow chart is shown in Supplementary Fig. 1.

This RCT was conducted in accordance with the protocol that was approved by the institutional review board of our hospital. All the candidates were well informed and provided their full consent after they received a verbal explanation of the study and an informational document $[16,17]$.

\section{Definitions}

The preoperative measurements of the complete blood counts (CBC), C-reactive protein (CRP) and albumin (ALB) were derived within the 7 days prior to surgery as stated previously [19]. All blood tests were performed using the same sample.

Neutrophil-lymphocyte ratio (NLR): the ratio of the number of neutrophils to the number of lymphocytes.
Platelet-lymphocyte ratio (PLR): the ratio of the number of platelets to the number of lymphocytes.

Systemic immune-inflammatory index (SII): SII: platelets $\times$ NLR.

C-reactive protein-albumin ratio (CAR): the serum CRP level divided by the ALB level.

Adjuvant chemotherapy: according to the patient's wishes and their physical condition, fluoride-based adjuvant chemotherapy was recommended for most patients with pathological stage II and III disease [20] in our center, as previously described [21,22]. A total of 256 patients (63.8\%) received adjuvant chemotherapy, with the median number of cycles being 5 (range 1-12), similar to a previous study [23]. According to the cycles they completed, the patients were divided into the following three groups: group A: patients who did not receive any chemotherapy treatment; group B: patients who completed 1-3 cycles of chemotherapy; and group C: patients who completed more than three cycles of chemotherapy [24].

Recurrence: recurrence was diagnosed based on radiologic findings or biopsies with suspicious lesions. The recurrence-free survival (RFS) period was defined as the period from the date of the surgery to the date of recurrence or last follow-up without recurrence. For RFS, the patients who died without known tumor recurrence were censored at the last documented evaluation. The recurrence patterns were classified as local recurrence (LR) (anastomotic or gastric remnants), lymph node (LN) and distant metastasis (DM) (peritoneal, hepatic, pulmonary, or other sites of metastatic disease) $[6,19,25]$. Post-recurrence survival (PRS) was defined as the period from the first recurrence to either death or the last follow-up. When patients were diagnosed with recurrence, systemic chemotherapy or supportive therapy were usually recommended according to the patient's willingness after a discussion with the multidisciplinary team (MDT).

\section{Follow-up}

The median follow-up time was 29 months (range 3-41 months). Overall survival (OS) was defined as the period from the date of surgery to the date of death or final follow-up, as previously described [26]. Postoperative follow-ups were performed every 3 months for 2 years and then every 6 months from years 3 to 5 . Most routine patient follow-up appointments included a physical examination, laboratory tests, chest radiography, abdominal ultrasonography or CT and an annual endoscopic examination.

\section{Statistical analysis}

Continuous variables are reported as the mean values \pm SD or median (interquartile range). Continuous data were 
compared across cohorts using an unpaired $t$ test unless the data were not normally distributed (as assessed by the Kolmogorov-Smirnov test). In these instances, the Mann-Whitney $U$ test was used. Categorical data were compared using the Chi square test or Fisher exact test wherever appropriate. Receiver operating characteristic (ROC) curves were generated to estimate the optimal threshold for NLR, SII, PLR and CAR as risk factors for early recurrence. The Akaike information criterion (AIC) asymptotically selects the model that minimizes the mean squared error of prediction or estimation [27]. The posterior model probabilities, defined by the possible subsets of the risk factors, can be approximated using the Bayesian information criterion (BIC), a simple expression that involves only the maximum likelihood, the sample size, and the number of risk factors in the model [28]. We used both the AIC and BIC to compare the predictive value of the different models. The concordance index ( $C$-index) was calculated to evaluate the discriminative ability of the different models [19]. RFS was assessed using the Kaplan-Meier method. A minimum- $p$ value approach was used to evaluate the optimal threshold of RFS to divide the patients into early- and late-recurrence cohorts based on the duration of PRS [8]. A Cox proportional hazards regression model was used to identify the independent prognostic factors associated with RFS. Variables with a value of $p<0.05$ in the univariate analysis were subsequently included in a multivariate analysis. In addition, we applied decision curve analysis, which evaluates the clinical utility of a prediction model by calculating its net benefit using the rate of true and false positives in varied risk thresholds for screening [29]. Statistical analyses were performed using SPSS v.18.0 for Windows (SPSS Inc., Chicago, IL, USA) and R (https://www.r-project.org/). $p$ values less than 0.05 were considered statistically significant.

\section{Results}

\section{Clinicopathological characteristics of the patients}

The clinicopathological characteristics of the entire study population, which were stratified into patients with and without recurrence, are summarized in Table 1. At the time of the last follow-up, $96(23.9 \%)$ of the 401 patients had recurrence after gastrectomy. Compared with patients without recurrence, patients with recurrence exhibited poorer clinical features, such as higher tumor stage, larger tumor size and lymphovascular invasion (all $p<0.05$ ). The median RFS, PRS and overall survival (OS) of the patients with recurrence were 12 (95\% CI 10.8-12.6), 6 (95\% CI 4.4-7.6), and 21 (95\% CI 17.5-24.5) months, respectively.

\section{Defining early and late recurrence}

The evaluated early recurrence cutoff values and the associated survival outcomes, including the RFS, PRS, and OS, are shown in Table 2. In the present study population, of the 96 patients with recurrence, the optimal length of RFS to distinguish between early and late recurrence, based on the subsequent PRS, was 12 months $\left(p=1.1 \times 10^{-5}\right)$ (Fig. 1). The median RFS in the early ( $<12$ months) recurrence group $(n=44,45.8 \%)$ was 7.0 months (95\% CI 4.8-9.2), which was followed by a relatively limited PRS of 3.5 months (95\% CI 2.5-4.5) (Supplementary Fig. 2). The patients with recurrence after 12 months $(n=52,54.2 \%)$ had a median RFS of 21.0 months (95\% CI 18.5-23.5), with a median PRS of 9.5 months (95\% CI 7.8-11.2). The median OS was significantly longer for the patients with late recurrence (32.0 months, 95\% CI 27.7-36.3) than for the patients with early recurrence (13.0 months, 95\% CI 11.8-14.1, $p<0.001)$. The multivariate analysis revealed that early recurrence (HR 3.221, 95\% CI 1.821-5.663) was an independent risk factor for PRS in patients with recurrence, independent of treatment after recurrence (HR 0.418, 95\% CI 0.254-0.688) (Supplementary Table 1).

\section{Clinicopathological characteristics of the patients with recurrence}

The patients with early recurrence more often had a larger tumor size (61.3 vs $51.8 \mathrm{~mm}, p=0.032)$ and higher levels of inflammatory indices, while patients with late recurrence more frequently received adjuvant chemotherapy $(p<0.001)$. There was no difference between the early recurrence and late recurrence groups in the observed recurrence patterns, including distant metastasis (36 vs $38, p=0.310$ ), local recurrence ( 6 vs $9, p=0.622)$ and lymph nodal recurrence ( 15 vs $21, p=0.526$ ) (Table 3 ). In addition, no significant differences were observed in treatment after recurrence between the early recurrence and late recurrence groups $(p=0.184)$.

\section{ROC curves and optimal cutoff values of the preoperative inflammatory indices for early recurrence}

Supplementary Fig. 3 shows the ROC curves of four different preoperative inflammatory indices, including SII, NLR, PLR, and CAR. The area under the curve (AUC) values was $0.707,0.651,0.672$, and 0.725 , respectively (all $p<0.05$ ) (Supplementary Table 2). The responding optimal cutoff 
Table 1 Clinicopathological characteristics of all the patients

\begin{tabular}{|c|c|c|c|c|}
\hline & All patients $(n=401)$ & No recurrence $(n=305)$ & Recurrence $(n=96)$ & $p$ value \\
\hline Age, mean years $(\mathrm{SD})$ & $58.6(10.3)$ & $58.1(10.5)$ & $60.3(9.3)$ & 0.054 \\
\hline Sex, $n(\%)$ & & & & 0.303 \\
\hline Male & $271(67.6 \%)$ & $202(66.2 \%)$ & $69(71.9 \%)$ & \\
\hline Female & $130(32.4 \%)$ & $103(33.8 \%)$ & $27(28.1 \%)$ & \\
\hline Tumor diameter, mean, mm (SD) & $41.5(23.1)$ & $36.8(21.6)$ & $56.1(21.6)$ & $<0.001$ \\
\hline Tumor location, $n(\%)$ & & & & 0.035 \\
\hline Upper & $117(29.2 \%)$ & $78(25.6 \%)$ & $39(40.6 \%)$ & \\
\hline Middle & $69(17.2 \%)$ & $57(18.7 \%)$ & $12(12.5 \%)$ & \\
\hline Lower & $191(47.6 \%)$ & $152(49.8 \%)$ & $39(40.6 \%)$ & \\
\hline Mix & $24(6.0 \%)$ & $18(5.9 \%)$ & $6(6.3 \%)$ & \\
\hline Gastrectomy extent, $n(\%)$ & & & & 0.004 \\
\hline Distal & $168(41.9 \%)$ & $140(45.9 \%)$ & $28(29.2 \%)$ & \\
\hline Total & $233(58.1 \%)$ & $165(54.1 \%)$ & $68(70.8 \%)$ & \\
\hline Reconstruction & & & & 0.004 \\
\hline B-I & $84(20.9 \%)$ & $75(24.6 \%)$ & $9(9.4 \%)$ & \\
\hline B-II & $84(20.9 \%)$ & $64(21.0 \%)$ & $20(20.8 \%)$ & \\
\hline Roux-en-Y & $233(58.1 \%)$ & $166(54.4 \%)$ & $67(69.8 \%)$ & \\
\hline Pathological type, $n(\%)$ & & & & 0.001 \\
\hline Differentiated & $168(41.9 \%)$ & $142(46.6 \%)$ & $26(27.1 \%)$ & \\
\hline Undifferentiated & $233(58.1 \%)$ & $163(53.4 \%)$ & $70(72.9 \%)$ & \\
\hline Lymphovascular invasion, $n(\%)$ & & & & $<0.001$ \\
\hline No & $228(56.9 \%)$ & $208(68.2 \%)$ & $20(20.8 \%)$ & \\
\hline Yes & $173(43.1 \%)$ & $97(31.8 \%)$ & $76(79.2 \%)$ & \\
\hline \multicolumn{5}{|l|}{ Preop SII } \\
\hline Median (IQR) & $579(372-835)$ & $551(349-764)$ & $749(464-1044)$ & 0.001 \\
\hline \multicolumn{5}{|l|}{ Preop NLR } \\
\hline Median (IQR) & $2.2(1.6-3.1)$ & $2.2(1.5-3.0)$ & $2.4(1.8-3.8)$ & 0.004 \\
\hline \multicolumn{5}{|l|}{ Preop PLR } \\
\hline Median (IQR) & $142(105-192)$ & $139(103-183)$ & $152(122-214)$ & 0.008 \\
\hline \multicolumn{5}{|l|}{ Preop CRP/ALB } \\
\hline Median (IQR) & $0.072(0.044-0.127)$ & $0.065(0.040-0.110)$ & $0.105(0.063-0.167)$ & $<0.001$ \\
\hline Adjuvant chemotherapy, $n(\%)$ & & & & $<0.001$ \\
\hline No & $145(36.2 \%)$ & $125(41.0 \%)$ & $20(20.8 \%)$ & \\
\hline $1-3$ cycles & $96(23.9 \%)$ & $59(19.3 \%)$ & $37(38.5 \%)$ & \\
\hline$>3$ cycles & $160(39.9 \%)$ & $121(39.7 \%)$ & $39(40.6 \%)$ & \\
\hline pT stage, $n(\%)$ & & & & $<0.001$ \\
\hline $\mathrm{T} 1$ & $125(31.2 \%)$ & $121(39.7 \%)$ & $4(4.2 \%)$ & \\
\hline $\mathrm{T} 2$ & $43(10.7 \%)$ & $40(13.1 \%)$ & $3(3.1 \%)$ & \\
\hline $\mathrm{T} 3$ & $127(31.7 \%)$ & $96(31.5 \%)$ & $31(32.3 \%)$ & \\
\hline $\mathrm{T} 4$ & $106(26.4 \%)$ & $48(15.7 \%)$ & $58(60.4 \%)$ & \\
\hline pN stage, $n(\%)$ & & & & $<0.001$ \\
\hline No & $158(39.4 \%)$ & $154(50.5 \%)$ & $4(4.2 \%)$ & \\
\hline N1 & $61(15.2 \%)$ & $51(16.7 \%)$ & $10(10.4 \%)$ & \\
\hline $\mathrm{N} 2$ & $66(16.5 \%)$ & $50(16.4 \%)$ & $16(16.7 \%)$ & \\
\hline N3 & $116(28.9 \%)$ & $50(16.4 \%)$ & $66(68.8 \%)$ & \\
\hline pTNM, $n(\%)$ & & & & $<0.001$ \\
\hline I & $135(33.7 \%)$ & $132(43.3 \%)$ & $3(3.1 \%)$ & \\
\hline II & $84(20.9 \%)$ & $78(25.6 \%)$ & $6(6.3 \%)$ & \\
\hline III & $182(45.4 \%)$ & $95(31.1 \%)$ & $87(90.6 \%)$ & \\
\hline Survival (median months) & & & & \\
\hline
\end{tabular}


Table 1 (continued)

\begin{tabular}{|c|c|c|c|c|}
\hline & All patients $(n=401)$ & No recurrence $(n=305)$ & Recurrence $(n=96)$ & $p$ value \\
\hline Recurrence-free survival & NA & NA & $12(9.1-14.9)$ & NA \\
\hline Post-recurrence survival & NA & NA & $6(4.4-7.6)$ & NA \\
\hline Overall survival & NA & NA & $21(17.5-24.5)$ & NA \\
\hline Death, $n(\%)$ & & & & $<0.001$ \\
\hline No & $323(80.5 \%)$ & $298(97.7 \%)$ & $25(26.0 \%)$ & \\
\hline Yes & $78(19.5 \%)$ & $7(2.3 \%)$ & $71(74.0 \%)$ & \\
\hline
\end{tabular}

$S D$ standard deviation, preop preoperative, $S I I$ systemic immune-inflammatory index, $N L R$ neutrophil-lymphocyte ratio, $P L R$ platelet-lymphocyte ratio, $C A R$ C-reactive protein-albumin ratio, $I Q R$ interquartile range, $N A$ not applicable

Table 2 Evaluated cutoff thresholds for defining early and late recurrence based on the prognosis after recurrence

\begin{tabular}{|c|c|c|c|c|c|c|c|c|c|}
\hline \multirow{2}{*}{$\begin{array}{l}\text { Evaluated } \\
\text { cutoff }(<)\end{array}$} & \multirow[t]{2}{*}{$p$ value } & \multicolumn{4}{|c|}{ Potential early-recurrence cohort } & \multicolumn{4}{|c|}{ Potential late-recurrence cohort } \\
\hline & & $N$ & RFS (months) & PRS (months) & OS (months) & $N$ & RFS (months) & PRS (months) & OS (months) \\
\hline 6 & 0.051 & 15 & 3.5 & 2.0 & 7.0 & 81 & 16.0 & 6.0 & 23.5 \\
\hline 7 & 0.003054 & 19 & 4.5 & 2.0 & 7.0 & 77 & 17.0 & 7.0 & 24.0 \\
\hline 8 & 0.005792 & 23 & 5.0 & 2.0 & 9.0 & 73 & 17.5 & 7.0 & 24.0 \\
\hline 9 & 0.020081 & 25 & 5.0 & 2.0 & 9.0 & 71 & 18.0 & 7.0 & 24.0 \\
\hline 10 & 0.009682 & 28 & 5.0 & 3.0 & 10.0 & 68 & 18.0 & 7.0 & 26.0 \\
\hline 11 & 0.005396 & 31 & 6.0 & 3.0 & 10.0 & 65 & 18.0 & 7.0 & 27.0 \\
\hline 12 & 0.000011 & 44 & 7.0 & 3.5 & 13.0 & 52 & 21.0 & 9.5 & 32.0 \\
\hline 13 & 0.000223 & 49 & 8.0 & 4.0 & 14.0 & 47 & 21.5 & 10.0 & 32.0 \\
\hline 14 & 0.000117 & 50 & 8.0 & 4.0 & 14.0 & 46 & 21.5 & 11.0 & 33.0 \\
\hline 15 & 0.000023 & 53 & 9.0 & 4.0 & 14.0 & 43 & 22.0 & 11.0 & 34.0 \\
\hline 16 & 0.000023 & 53 & 9.0 & 4.0 & 14.0 & 43 & 22.0 & 11.0 & 34.0 \\
\hline 17 & 0.000028 & 57 & 10.0 & 4.0 & 14.0 & 39 & 22.0 & 11.0 & 36.0 \\
\hline 18 & 0.000052 & 60 & 10.0 & 4.0 & 14.0 & 36 & 22.0 & 11.0 & 36.0 \\
\hline 19 & 0.001266 & 64 & 10.5 & 4.0 & 15.0 & 32 & 23.0 & 11.0 & 36.0 \\
\hline 20 & 0.002868 & 66 & 10.5 & 4.0 & 15.0 & 30 & 24.0 & 11.0 & 36.0 \\
\hline 21 & 0.009494 & 69 & 11.0 & 5.0 & 16.0 & 27 & 24.0 & 11.0 & 36.0 \\
\hline 22 & 0.006673 & 72 & 11.0 & 5.0 & 16.0 & 24 & 25.0 & 11.0 & 36.0 \\
\hline 23 & 0.009546 & 78 & 11.0 & 5.0 & 17.0 & 18 & 26.0 & 11.0 & NA \\
\hline 24 & 0.020553 & 79 & 11.0 & 5.0 & 18.0 & 16 & 26.0 & 11.0 & NA \\
\hline
\end{tabular}

Shown in bold is the optimal cutoff threshold with the lowest $p$ value

$R F S$ recurrence-free survival, $P R S$ post-recurrence survival, $O S$ overall survival, $N A$ not applicable

values of the inflammatory indices for early recurrence were $599,3.1,198$, and 0.131 , respectively.

\section{Univariate and multivariate analyses of the factors associated with early recurrence}

The univariate analysis of early recurrence in the whole cohort revealed that age, tumor size, pTNM stage, differentiation type, lymphovascular invasion, reconstruction methods, adjuvant chemotherapy, SII, NLR, PLR, and CAR were related to early recurrence. Further multivariate analyses revealed that CAR (HR 2.108, $p=0.028$ ), pathological stage III (HR 10.560, $p=0.001)$, and adjuvant chemotherapy $>3$ cycles (HR 0.151, $p<0.001$ ) were independent predictive factors for early recurrence (Table 4).

\section{Incorporation of preoperative CAR levels into PTNM stage}

By combining the preoperative CAR and pTNM stage, a new prognostic predictive model (model B) was established according to the results of multivariate analyses. When compared with the predictive system without CAR (model A), model B had a lower AIC (456.6 vs 480.3) and BIC (464.6 vs 484.3$)$ and a higher C-index $(0.82,95 \%$ CI $0.77-0.87$ vs $0.75,95 \%$ CI $0.70-0.79, p<0.001)$. 
Fig. 1 Different cutoff thresholds, with the corresponding $p$ values, show that the optimal threshold for defining early and late recurrence based on the difference in post-recurrence survival is 12 months

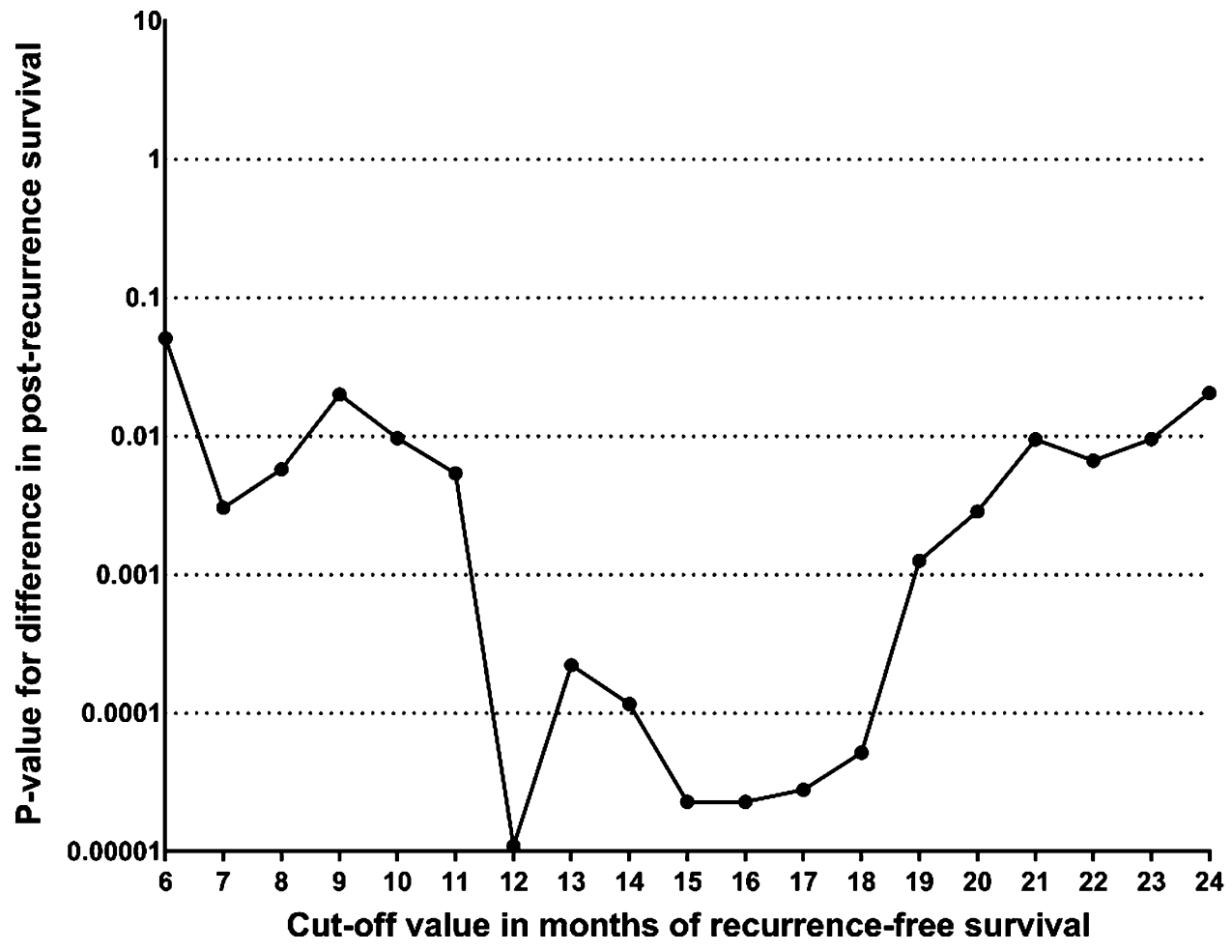

\section{Comparison of the clinical utility between the PTNM stage and the predictive model with CAR}

As shown in Fig. 2, we compared the net benefit of model B (composed of TNM stage with CAR) to model A (composed of only TNM stage) and demonstrated that in a wide range of threshold probabilities (1-70\%), the clinical net benefit of the former was larger than that of the latter.

\section{Effect of preoperative CAR on adjuvant chemotherapy in stage III patients}

Based on the results of the multivariate analysis, we further investigated the effect of CAR on adjuvant chemotherapy in patients with stage III gastric cancer. According to the cycles of postoperative adjuvant chemotherapy (PAC), the stage III patients were divided into three groups as follows: group A: without PAC; group B: received 1-3 cycles of PAC; and group C: PAC $>3$ cycles. Supplementary Table 3 shows the clinicopathological data from these three groups of patients. Figure $3 \mathrm{~A}$ shows the early recurrence rate of the three groups of patients, of which group A had the same early recurrence rate as group B (A vs B: $53.8 \%$ vs $32.5 \%$, $p=0.090$ ). The differences between the other groups were statistically significant (B vs C: $32.5 \%$ vs $7.2 \%, p<0.001$, and A vs C: $53.8 \%$ vs $7.2 \%, p<0.001)$. To explain the similar early recurrence rate between group $\mathrm{A}$ and group $\mathrm{B}$, all patients in stage III were further divided into the CAR $<0.131$ (LCAR) group and the CAR $>0.131$ (HCAR) group according to the optimal CAR cutoff value. Further stratified analysis revealed that for patients with pathological stage III and LCAR, the risk of early recurrence was significantly lower in group B (17.3\% vs $50.0 \%, p=0.029)$ and group $\mathrm{C}(4.2 \%$ vs $50.0 \%, p<0.001)$ than in group A. For patients with pathological stage III and HCAR, $>3$ cycles of PAC significantly reduced the risk of early recurrence compared with those without PAC ( $16.0 \%$ vs $54.2 \%, p=0.004)$, while the early recurrence rate of patients receiving 1-3 cycles of PAC was similar to that of patients without PAC $(58.3 \%$ vs $54.2 \%, p=0.824)$ (Fig. 3B-C).

\section{Discussion}

Previous studies have shown that patients who suffer the early recurrence of a variety of malignancies have a poor prognosis. However, no evidence-based definition exists for the early recurrence of GC after radical gastrectomy. The results of this study indicated that the optimal cutoff value to differentiate between early and late recurrence, based on PRS, was a recurrence-free interval of at least 12 months. In addition, we also explored the independent risk factors associated with early recurrence, including preoperative CAR $\geq 0.131$, pathological stage III, and PAC $>3$ cycles, which were independent protective factors against early recurrence. Moreover, the use of TNM stage in combination with the preoperative CAR level improved the predictive ability and clinical utility of the model over the use of 
Table 3 Clinicopathological characteristics of the patients with recurrence

\begin{tabular}{|c|c|c|c|}
\hline & Early recurrence $(n=44)$ & Late recurrence $(n=52)$ & $p$ value \\
\hline Age, mean years $(\mathrm{SD})$ & $62.0(9.1)$ & $58.9(9.3)$ & 0.103 \\
\hline Sex, $n(\%)$ & & & 0.864 \\
\hline Male & $12(27.3 \%)$ & $15(28.8 \%)$ & \\
\hline Female & $32(72.7 \%)$ & $37(71.2 \%)$ & \\
\hline Tumor diameter, mean, mm (SD) & $61.3(25.4)$ & $51.8(16.8)$ & 0.032 \\
\hline Tumor location, $n(\%)$ & & & 0.166 \\
\hline Upper & $15(34.1 \%)$ & $24(46.2 \%)$ & \\
\hline Middle & $7(15.6 \%)$ & $5(9.6 \%)$ & \\
\hline Lower & $17(38.6 \%)$ & $22(42.3 \%)$ & \\
\hline Mix & $5(11.4 \%)$ & $1(1.9 \%)$ & \\
\hline Gastrectomy extent, $n(\%)$ & & & 0.599 \\
\hline Distal & $14(31.8 \%)$ & $14(26.9 \%)$ & \\
\hline Total & $30(68.2 \%)$ & $38(73.1 \%)$ & \\
\hline Reconstruction & & & 0.323 \\
\hline B-I & $2(4.5 \%)$ & $7(13.5 \%)$ & \\
\hline B-II & $10(22.7 \%)$ & $10(19.2 \%)$ & \\
\hline Roux-en-Y & $32(72.7 \%)$ & $32(72.7 \%)$ & \\
\hline Pathological type, $n(\%)$ & & & 0.673 \\
\hline Differentiated & $11(25.0 \%)$ & $15(28.8 \%)$ & \\
\hline Undifferentiated & $33(75.0 \%)$ & $37(71.2 \%)$ & \\
\hline Lymphovascular invasion, $n(\%)$ & & & 0.153 \\
\hline No & $12(27.3 \%)$ & $8(15.4 \%)$ & \\
\hline Yes & $32(72.7 \%)$ & $44(84.6 \%)$ & \\
\hline \multicolumn{4}{|l|}{ Preop SII } \\
\hline Median (IQR) & $817(610-1327)$ & $645(397-913)$ & 0.015 \\
\hline \multicolumn{4}{|l|}{ Preop NLR } \\
\hline Median (IQR) & $2.9(2.1-4.0)$ & $2.3(1.7-2.9)$ & 0.048 \\
\hline \multicolumn{4}{|l|}{ Preop PLR } \\
\hline Median (IQR) & $175(136-265)$ & $142(106-186)$ & 0.007 \\
\hline \multicolumn{4}{|l|}{ Preop CRP/ALB } \\
\hline Median (IQR) & $0.148(0.070-0.255)$ & $0.091(0.048-0.130)$ & 0.005 \\
\hline Adjuvant chemotherapy, $n(\%)$ & & & $<0.001$ \\
\hline No & $15(34.1 \%)$ & $5(9.6 \%)$ & \\
\hline $1-3$ cycles & $22(50.0 \%)$ & $15(28.8 \%)$ & \\
\hline$>3$ cycles & $7(15.9 \%)$ & $32(61.5 \%)$ & \\
\hline pT stage, $n(\%)$ & & & 0.540 \\
\hline $\mathrm{T} 1$ & $3(6.8 \%)$ & $1(1.9 \%)$ & \\
\hline $\mathrm{T} 2$ & $1(2.3 \%)$ & $2(3.8 \%)$ & \\
\hline $\mathrm{T} 3$ & $12(27.3 \%)$ & $19(36.5 \%)$ & \\
\hline $\mathrm{T} 4$ & $28(63.6 \%)$ & $30(57.7 \%)$ & \\
\hline pN stage, $n(\%)$ & & & 0.457 \\
\hline No & $1(2.3 \%)$ & $3(5.8 \%)$ & \\
\hline $\mathrm{N} 1$ & $5(11.4 \%)$ & $5(9.6 \%)$ & \\
\hline $\mathrm{N} 2$ & $5(11.4 \%)$ & $11(21.2 \%)$ & \\
\hline N3 & $33(75.0 \%)$ & $33(63.5 \%)$ & \\
\hline pTNM stage, $n(\%)$ & & & 0.651 \\
\hline I & $2(4.5 \%)$ & $1(1.9 \%)$ & \\
\hline II & $2(4.5 \%)$ & $4(7.7 \%)$ & \\
\hline III & $40(90.6 \%)$ & $47(90.4 \%)$ & \\
\hline Treatment after recurrence & & & 0.184 \\
\hline Absent & $22(50 \%)$ & $19(36.5 \%)$ & \\
\hline
\end{tabular}


Table 3 (continued)

\begin{tabular}{lccc}
\hline & Early recurrence $(n=44)$ & Late recurrence $(n=52)$ & $p$ value \\
\hline Present & $22(50 \%)$ & $33(63.5 \%)$ & \\
Recurrence patterns & & & \\
Distant metastasis, $n(\%)$ & $36(81.8 \%)$ & $38(73.1 \%)$ & 0.310 \\
Local recurrence, $n(\%)$ & $6(13.6 \%)$ & $9(17.3 \%)$ & 0.622 \\
Lymph nodal recurrence, $n(\%)$ & $15(34.1 \%)$ & $21(40.4 \%)$ & 0.526 \\
\hline
\end{tabular}

$S D$ standard deviation; preop, preoperative, SII systemic immune-inflammatory index, NLR neutrophillymphocyte ratio, $P L R$ platelet-lymphocyte ratio, $C A R$ C-reactive protein-albumin ratio, IQR interquartile range, $N A$ not applicable

TNM stage alone. Finally, the stratified analysis revealed that $C A R \geq 0.131$ affected the efficacy of PAC in stage III GC patients. In patients with stage III disease and a preoperative CAR $\geq 0.131,1-3$ cycles of PAC did not exert a protective effect against early recurrence.

Throughout the literature, the definition of early recurrence after radical gastrectomy differs greatly. Eom and Ogata et al. defined early recurrence as recurrent disease within 1 year after surgery $[13,30]$. Otsuji, Kang, Lai and Shiraishi et al. defined early recurrence as death from tumor recurrence within 2 years after surgery $[12,14,15$, 31]. Adachi et al. defined it as death from tumor recurrence within 3 years after surgery [32]. However, neither PRS outcomes nor potential differences between the two patient populations are mentioned. The use of OS or DFS as the primary outcome may be biased in determining early and late recurrence because patients with late recurrence experience a long period without recurrence, and thus, their OS and DFS are expected to be longer. To avoid this bias, this study, for the first time in the field of GC, used PRS as the outcome indicator and found that early recurrence should be defined as recurrence within 12 months after radical gastrectomy.

Since Virchow first discovered the relationship between inflammation and cancer [33], increasingly more evidence shows that tumor progression is not only related to the intrinsic properties of the tumor cells but also inseparable from the body's inflammatory immune response [34]. A series of studies confirm that inflammatory indices such as NLR and CAR are closely related to the OS of gastric cancer [35-37]. However, few studies have focused on the predictive ability of inflammatory indices for postoperative recurrence, especially for early recurrence. In the present study, preoperative NLR, SII, PLR, and CAR were simultaneously included in the multivariate analysis, and only CAR (HR 2.108, 95\% CI 1.082-4.107) was an independent risk factor for early recurrence, with the highest AUC value (0.725, sensitivity 55.8\% and specificity $80.4 \%$ ). Due to the high specificity of CAR, we recommend that physicians treating patients with preoperative $\mathrm{CAR} \geq 0.131$ adopt a more positive follow-up strategy with these patients. In addition, the predictive model B (composed of TNM stage with CAR) had a lower AIC and
BIC and a higher $C$-index than model A (composed of only TNM stage), which suggested that model B had a good predictive ability for early recurrence compared to model A. In addition, we performed a decision curve analysis to evaluate the clinical utility of model A and model B by calculating the net benefit in various risk thresholds for screening. We found that in a wide range of threshold probabilities (1-70\%), the curve of model B was always higher than that of model A, which implied that if we used a risk threshold from the given interval (e.g., 30\%), screening should be recommended. Moreover, if an individual's risk was above the given threshold, then the calculated net benefit (the weighted sum of true positives subtracted by the number of false positives) was larger for model B than for model A.

Many studies have confirmed that PAC significantly prolongs RFS in patients with gastric cancer after radical gastrectomy [38, 39]. However, whether PAC effectively reduces the early recurrence rate has not been reported. The multivariate analysis of the whole cohort revealed that PAC with $>3$ cycles (HR $0.151,95 \%$ CI $0.058-0.393$ ) effectively reduced the early recurrence rate. However, 1-3 cycles of PAC did not exert a protective effect against early recurrence. To explain this finding, according to the results of the multivariate analysis as well as the number of early recurrence cases, we further stratified the patients with pathologic stage III and found that, compared with the patients without PAC, the patients with preoperative CAR $\geq 0.131 \mathrm{did}$ not benefit from 1 to 3 cycles of PAC. In the present study, for the first time, we found preoperative CAR to be a novel and promising predictor of PAC response, which may help physicians identify a subset of patients with GC who might benefit from adjuvant therapy after surgery. However, the molecular biological mechanism has not yet been elucidated. Compared with the predictive single-patient classifier test for chemotherapy found by Cheong [40] and the microsatellite instability proposed by An [41] and Choi [42], CAR is easily detected from routinely performed, inexpensive blood tests without the need for additional specimens.

The present study has several limitations. First, this was an exploratory study conducted in a single center with a small sample size, which lacks external validation. Second, basic 
Table 4 Univariate and multivariate analyses of the factors associated with early recurrence

\begin{tabular}{|c|c|c|c|c|c|c|}
\hline & \multicolumn{3}{|c|}{ Univariate analysis } & \multicolumn{3}{|c|}{ Multivariate analysis } \\
\hline & HR & $95 \% \mathrm{CI}$ & $p$ value & HR & $95 \% \mathrm{CI}$ & $p$ value \\
\hline \multicolumn{7}{|l|}{ Sex } \\
\hline Female & 1.000 & & & & & \\
\hline Male & 1.304 & $0.672-2.533$ & 0.432 & & & \\
\hline \multicolumn{7}{|l|}{ Age (years) } \\
\hline$<65$ & 1.000 & & & 1.000 & & \\
\hline$\geq 65$ & 2.214 & $1.226-3.998$ & 0.008 & 1.149 & $0.588-2.246$ & 0.685 \\
\hline \multicolumn{7}{|c|}{ Tumor diameter (mm) } \\
\hline$<50$ & 1.000 & & & 1.000 & & \\
\hline$\geq 50$ & 5.208 & $2.631-10.306$ & $<0.001$ & 1.642 & $0.756-3.570$ & 0.210 \\
\hline \multicolumn{7}{|l|}{ Tumor location } \\
\hline Upper & 1.000 & & & & & \\
\hline Middle & 0.769 & $0.314-1.886$ & 0.567 & & & \\
\hline Lower & 0.690 & $0.344-1.381$ & 0.294 & & & \\
\hline Mix & 1.027 & $0.373-2.827$ & 0.958 & & & \\
\hline \multicolumn{7}{|l|}{ Gastrectomy extent } \\
\hline Distal & 1.000 & & & & & \\
\hline Total & 1.582 & $0.839-2.983$ & 0.157 & & & \\
\hline \multicolumn{7}{|l|}{ Reconstruction } \\
\hline B-I & 1.000 & & & 1.000 & & \\
\hline B-II & 5.229 & $1.146-23.864$ & 0.033 & 2.971 & $0.615-14.354$ & 0.175 \\
\hline Roux-en-Y & 6.111 & $1.465-25.502$ & 0.013 & 1.758 & $0.396-7.808$ & 0.458 \\
\hline \multicolumn{7}{|l|}{ Pathological type } \\
\hline Differentiated & 1.000 & & & 1.000 & & \\
\hline Undifferentiated & 2.210 & $1.117-4.372$ & 0.023 & 1.968 & $0.942-4.112$ & 0.072 \\
\hline \multicolumn{7}{|c|}{ Lymphovascular invasion } \\
\hline No & 1.000 & & & 1.000 & & \\
\hline Yes & 3.770 & $1.942-7.321$ & $<0.001$ & 1.015 & $0.463-2.226$ & 0.969 \\
\hline \multicolumn{7}{|l|}{ SII } \\
\hline$<599$ & 1.000 & & & 1.000 & & \\
\hline$\geq 599$ & 4.887 & $2.349-10.169$ & $<0.001$ & 1.543 & $0.610-3.899$ & 0.360 \\
\hline \multicolumn{7}{|l|}{ NLR } \\
\hline$<3.1$ & 1.000 & & & 1.000 & & \\
\hline$\geq 3.1$ & 3.297 & $1.825-5.954$ & $<0.001$ & 2.003 & $0.949-4.229$ & 0.068 \\
\hline \multicolumn{7}{|l|}{ PLR } \\
\hline$<198$ & 1.000 & & & 1.000 & & \\
\hline$\geq 198$ & 2.999 & $1.657-5.430$ & $<0.001$ & 1.229 & $0.588-2.568$ & 0.584 \\
\hline \multicolumn{7}{|l|}{ CAR } \\
\hline$<0.131$ & 1.000 & & & 1.000 & & \\
\hline$\geq 0.131$ & 4.777 & $2.630-8.677$ & $<0.001$ & 2.108 & $1.082-4.107$ & 0.028 \\
\hline \multicolumn{7}{|c|}{ Adjuvant chemotherapy } \\
\hline No & 1.000 & & & 1.000 & & \\
\hline $1-3$ cycles & 2.386 & $1.238-4.600$ & 0.009 & 1.005 & $0.487-2.074$ & 0.989 \\
\hline$>3$ cycles & 0.409 & $0.167-1.004$ & 0.051 & 0.151 & $0.058-0.393$ & $<0.001$ \\
\hline \multicolumn{7}{|l|}{ pTNM stage } \\
\hline I & 1.000 & & & 1.000 & & \\
\hline II & 1.599 & $0.225-11.349$ & 0.639 & 1.202 & $0.154-9.363$ & 0.861 \\
\hline III & 16.592 & $4.009-68.663$ & $<0.001$ & 10.560 & $1.954-57.065$ & 0.006 \\
\hline
\end{tabular}

SII systemic immune-inflammatory index, NLR neutrophil-lymphocyte ratio, PLR platelet-lymphocyte ratio, $C A R$ C-reactive protein-albumin ratio 
Fig. 2 Decision curve analyses for the two models for early recurrence in patients with gastric cancer after radical gastrectomy

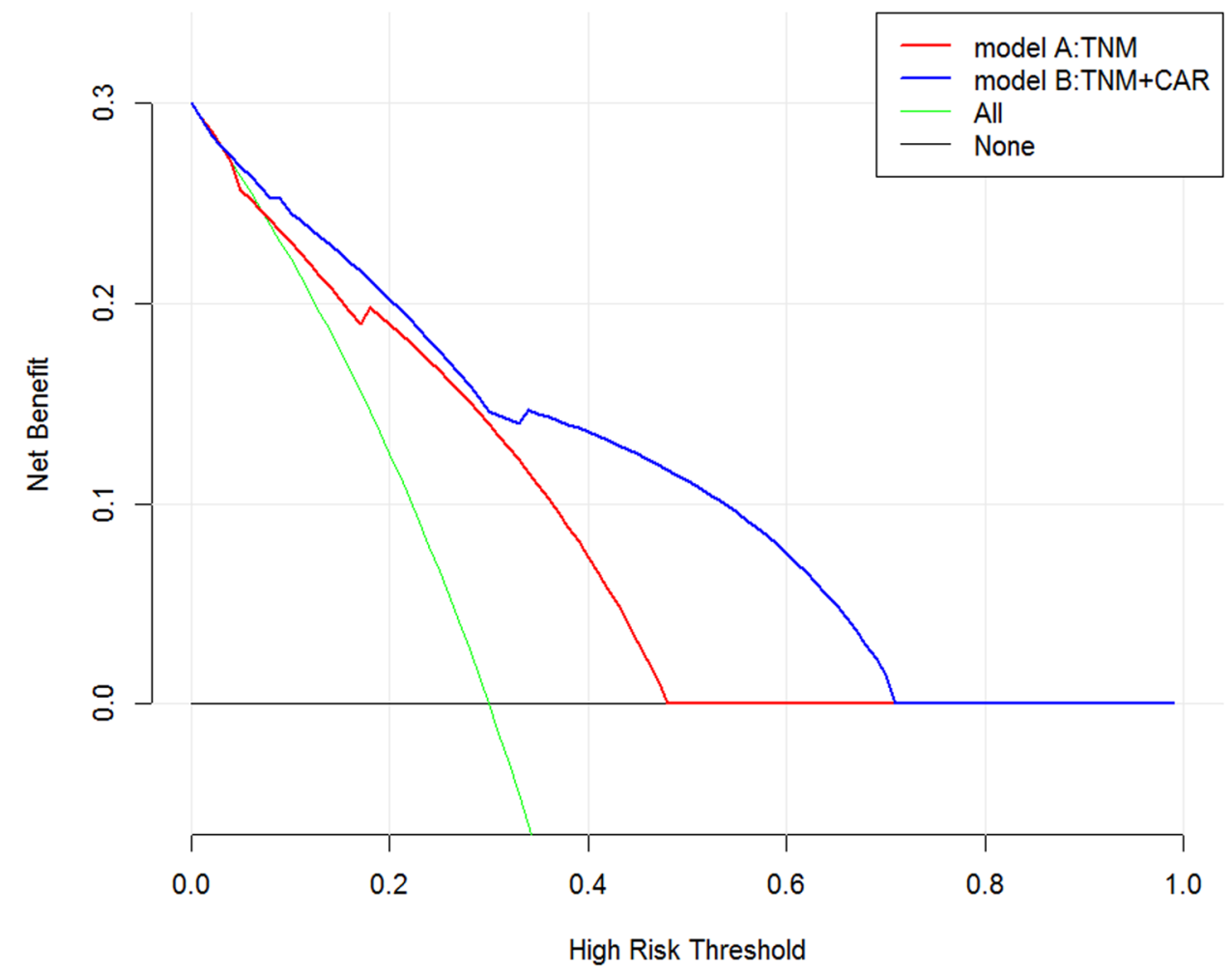

research is lacking to explore the detailed mechanism of how CAR is involved in the early recurrence and chemotherapy response of GC. Third, we did not analyze the relationship between neoadjuvant chemotherapy and early recurrence. Therefore, the conclusion may not be suitable for such patients. In addition, when patients were diagnosed with recurrence and after discussion with the MDT, some patients chose to receive treatment at the local hospital, so we did not have access to their detailed treatment regimens. Thus, the present study did not compare the detailed treatment regimens after recurrence between the early and late recurrence cohorts. Although the follow-up duration is short for our cohort, most patients with GC will experience recurrence within 2 years after radical gastrectomy [19]; thus, our results still have some clinical significance. To the best of our knowledge, the present study is the first to establish an evidence-based definition of early recurrence and to explore the independent predictors associated with early recurrence, which may help clinicians stratify GC patients and make more suitable treatment decisions. This work also provides a research direction for further investigations into the prognostic stratification of patients with recurrence and for exploring factors related to chemotherapy benefits.

\section{Conclusion}

In summary, the results of the present study indicated that a recurrence-free interval of 12 months is the optimal threshold for differentiating between early and late recurrence based on the subsequent prognosis. Compared with other inflammatory indices, the preoperative CAR level was the only independent risk factor for early recurrence. Moreover, the predictive ability of early recurrence and clinical utility were significantly higher when a combination of preoperative CAR and TNM stage was used than when only TNM stage was used. More importantly, patients with pathological stage III and preoperative CAR $<0.131$ benefited from PAC regardless of the number of cycles. However, 1-3 cycles of PAC may not exert a protective effect against early recurrence for patients with pathological stage III disease and preoperative CAR $\geq 0.131$. Appropriately designed prospective studies are needed to further confirm this hypothesis. 

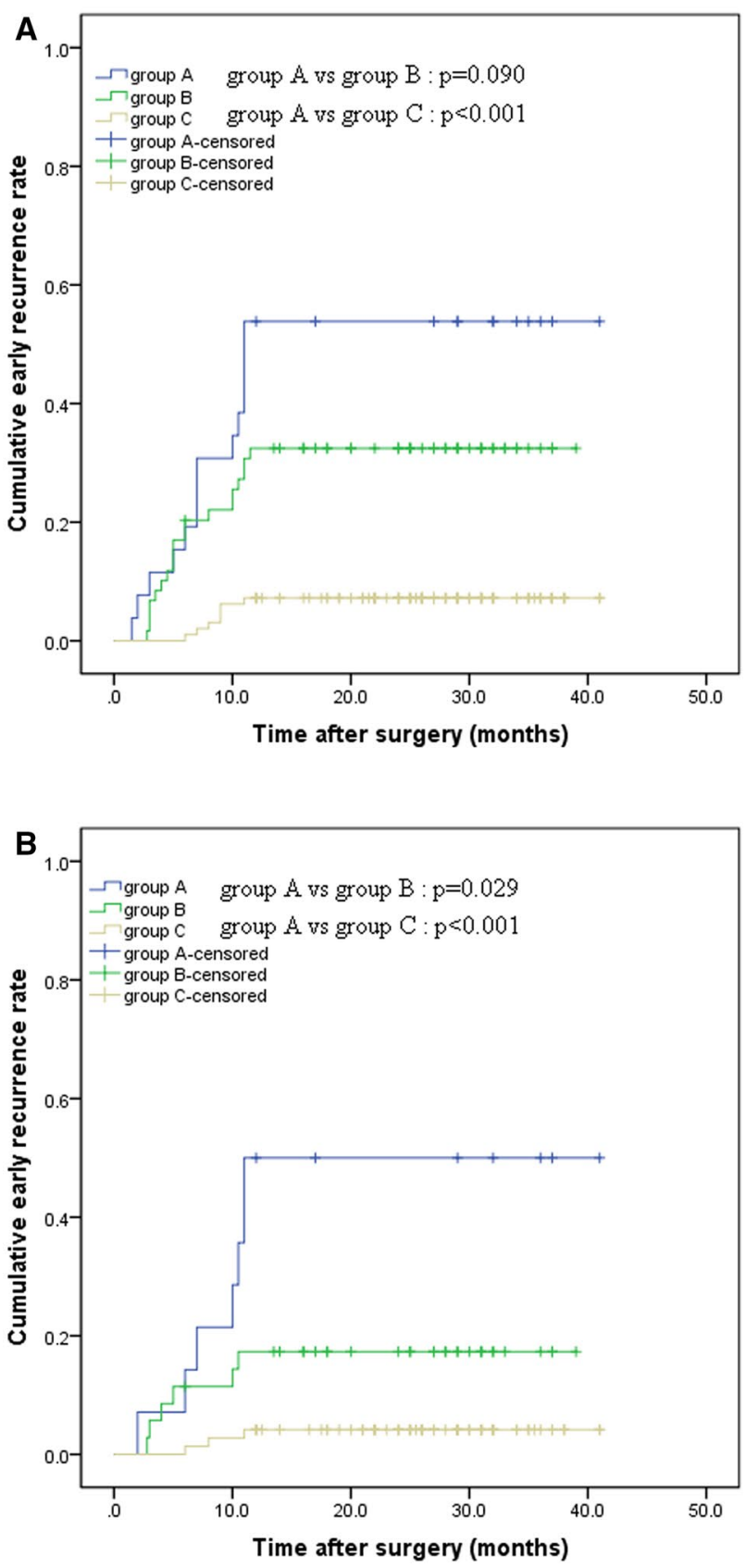

Fig. 3 Comparison of early recurrence rates in stage III GC patients after radical gastrectomy among different postoperative adjuvant chemotherapy (PAC) cycles. Group A: patients without PAC; group B: patients with 1-3 cycles of PAC; group C: patients with $>3$ cycles

Acknowledgements This study was funded by the Scientific and Technological Innovation Joint Capital Projects of Fujian Province (2016Y9031); the National Nature Science Foundation of China (no. 81871899); the Construction Project of Fujian Province Minimally Invasive Medical Center (no. [2017]171); the second batch of Special Support Funds for Fujian Province Innovation and Entrepreneurship Talents (2016B013); the QIHANG Fund of Fujian Medical University

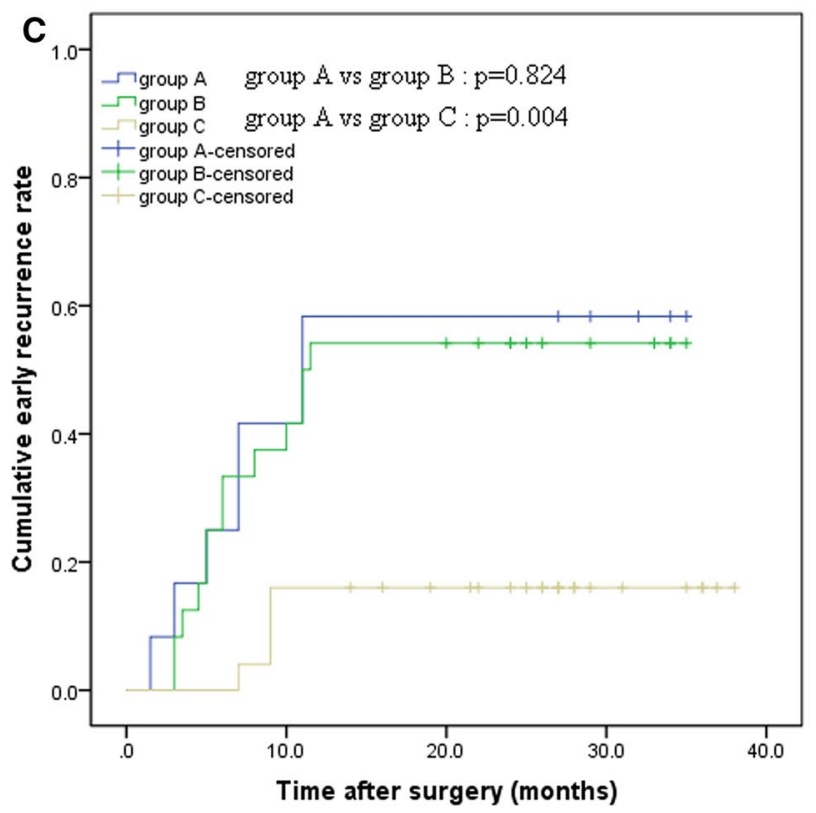

of PAC. a In the entire stage III cohort; $\mathbf{b}$ in patients with stage III and preoperative $\mathrm{C}$-reactive protein-albumin ratio $(\mathrm{CAR})<0.131$; $\mathbf{c}$ in patients with stage III and preoperative CAR $\geq 0.131$

(no. 2016QH025); the Fujian Province Medical Innovation Project (2015-CXB-16); the Fujian Provincial Health and Family Planning Commission Joint Project (WKJ2016-2-27); and the Chinese Physicians' Association Young Physician Respiratory Research Fund. 
Author contributions JL, BX, ZZ, CHZ and $\mathrm{CH}$ conceived the study, analyzed the data, and drafted the manuscript. CHZ, $\mathrm{CH}$ and $\mathrm{PL}$ helped critically revise the manuscript for important intellectual content. PL, JX, JBW, JXL, QYC, LLC, ML, RHT, ZNH and JL helped collect data and design the study.

\section{Compliance with ethical standards}

Conflict of interest All authors declare that there are no conflicts of interest or financial ties to disclose.

Human rights statement All procedures followed were in accordance with the ethical standards of the Responsible Committee on Human Experimentation (institutional and national) and with the 1964 Declaration of Helsinki and its later versions.

Informed consent Informed consent or a suitable substitute was obtained from all patients before being included in the study.

\section{References}

1. Fock KM. Review article: the epidemiology and prevention of gastric cancer. Aliment Pharmacol Ther. 2014;40(3):250-60.

2. Lin JP, Lin JX, Cao LL, et al. Preoperative lymphocyte-tomonocyte ratio as a strong predictor of survival and recurrence for gastric cancer after radical-intent surgery. Oncotarget. 2017;8(45):79234-47.

3. Chang JS, Kim KH, Yoon HI, et al. Locoregional relapse after gastrectomy with D2 lymphadenectomy for gastric cancer. Br J Surg. 2017;104(7):877-84.

4. Ito S, Ohashi Y, Sasako M. Survival after recurrence in patients with gastric cancer who receive $\mathrm{S}-1$ adjuvant chemotherapy: exploratory analysis of the ACTS-GC trial. BMC Cancer. 2018;18(1):449.

5. Lee JH, Chang KK, Yoon C, et al. Lauren histologic type is the most important factor associated with pattern of recurrence following resection of gastric adenocarcinoma. Ann Surg. 2018;267(1):105-13.

6. Lee D, Son SY, Kim YB, et al. Neural invasion is a significant contributor to peritoneal recurrence in signet ring cell gastric carcinoma. Ann Surg Oncol. 2018;25(5):1167-75.

7. Yamamoto Y, Ikoma H, Morimura R, et al. Optimal duration of the early and late recurrence of hepatocellular carcinoma after hepatectomy. World J Gastroenterol. 2015;21(4):1207-15.

8. Groot VP, Gemenetzis G, Blair AB, et al. Defining and predicting early recurrence in 957 patients with resected pancreatic ductal adenocarcinoma. Ann Surg. 2018. https://doi.org/10.1097/ SLA.0000000000002734.

9. Li T, Fan J, Qin LX, et al. Risk factors, prognosis, and management of early and late intrahepatic recurrence after resection of primary clear cell carcinoma of the liver. Ann Surg Oncol. 2011;18(7):1955-63.

10. Kiankhooy A, Taylor MD, LaPar DJ, et al. Predictors of early recurrence for node-negative $\mathrm{t} 1 \mathrm{to} \mathrm{t} 2 \mathrm{~b}$ non-small cell lung cancer. Ann Thorac Surg. 2014;98(4):1175-83.

11. Zhang XF, Beal EW, Bagante F, et al. Early versus late recurrence of intrahepatic cholangiocarcinoma after resection with curative intent. Br J Surg. 2018;105(7):848-56.

12. Otsuji E, Kobayashi S, Okamoto K, et al. Is timing of death from tumor recurrence predictable after curative resection for gastric cancer? World J Surg. 2001;25(11):1373-6.
13. Eom BW, Yoon H, Ryu KW, et al. Predictors of timing and patterns of recurrence after curative resection for gastric cancer. Dig Surg. 2010;27(6):481-6.

14. Kang WM, Meng QB, Yu JC, et al. Factors associated with early recurrence after curative surgery for gastric cancer. World J Gastroenterol. 2015;21(19):5934-40.

15. Lai JF, Kim S, Kim K, et al. Prediction of recurrence of early gastric cancer after curative resection. Ann Surg Oncol. 2009;16(7):1896-902.

16. Lu J, Zheng CH, Zheng HL, et al. Randomized, controlled trial comparing clinical outcomes of 3D and 2D laparoscopic surgery for gastric cancer: an interim report. Surg Endosc. 2017;31(7):2939-45.

17. Zheng $\mathrm{CH}$, Lu J, Zheng HL, et al. Comparison of 3D laparoscopic gastrectomy with a 2D procedure for gastric cancer: a phase 3 randomized controlled trial. Surgery. 2018;163(2):300-4.

18. Washington $\mathrm{K}$. 7th edition of the AJCC cancer staging manual: stomach. Ann Surg Oncol. 2010;17(12):3077-9.

19. Lu J, Xu BB, Zheng ZF, et al. CRP/prealbumin, a novel inflammatory index for predicting recurrence after radical resection in gastric cancer patients: post hoc analysis of a randomized phase III trial. Gastric Cancer. 2018. https://doi.org/10.1007/s1012 0-018-0892-0.

20. Sano T, Aiko T. New Japanese classifications and treatment guidelines for gastric cancer: revision concepts and major revised points. Gastric Cancer. 2011;14(2):97-100.

21. Zheng ZF, Lu J, Wang W, et al. Development and external validation of a simplified nomogram predicting individual survival after $\mathrm{R} 0$ resection for gastric cancer: an international, multicenter study. Ann Surg Oncol. 2018;25(8):2383-90.

22. Lu J, Zheng ZF, Wang W, et al. A novel TNM staging system for gastric cancer based on the metro-ticket paradigm: a comparative study with the AJCC-TNM staging system. Gastric Cancer. 2019. https://doi.org/10.1007/s10120-018-00904-w.

23. Chen S, Feng XY, Li YF, et al. The prognosis of gastric cancer patients with marginally elevated carcinoembryonic antigen (CEA) values after D2 radical gastrectomy. J Surg Oncol. 2013;107(6):641-5.

24. Fujitani K, Tamura S, Kimura Y, et al. Three-year outcomes of a phase II study of adjuvant chemotherapy with S-1 plus docetaxel for stage III gastric cancer after curative D2 gastrectomy. Gastric Cancer. 2013;17(2):348-53.

25. Jin LX, Moses LE, Squires MH 3rd, et al. Factors associated with recurrence and survival in lymph node-negative gastric adenocarcinoma: a 7-institution study of the US Gastric Cancer Collaborative. Ann Surg. 2015;262(6):999-1005.

26. Xu BB, Lu J, Zheng ZF, et al. Comparison of short-term and longterm efficacy of laparoscopic and open gastrectomy in high-risk patients with gastric cancer: a propensity score-matching analysis. Surg Endosc. 2018. https://doi.org/10.1007/s00464-018-6268-z.

27. Vrieze SI. Model selection and psychological theory: a discussion of the differences between the Akaike information criterion (AIC) and the Bayesian information criterion (BIC). Psychol Methods. 2012;17(2):228-43.

28. Raftery AE. Bayesian information criterion for censored survival models.\%A Volinsky CT. Biometrics. 2000;56(1):256-62.

29. Vickers AJ, Elkin EB. Decision curve analysis: a novel method for evaluating prediction models. Med Decis Making. 2006;26(6):565-74.

30. Ogata K, Mochiki E, Yanai M, et al. Factors correlated with early and late recurrence after curative gastrectomy for gastric cancer. Hepatogastroenterology. 2009;56(96):1760-4.

31. Shiraishi N, Inomata M, Osawa N, et al. Early and late recurrence after gastrectomy for gastric carcinoma. Univariate and multivariate analyses. Cancer. 2000;89(2):255-61. 
32. Adachi Y, Oshiro T, Mori M, et al. Prediction of early and late recurrence after curative resection for gastric carcinoma. Cancer. 1996;77(12):2445-8.

33. Balkwill F, Mantovani A. Inflammation and cancer: back to Virchow? Lancet. 2001;357(9255):539-45.

34. Alifano M, Mansuet-Lupo A, Lococo F, et al. Systemic inflammation, nutritional status and tumor immune microenvironment determine outcome of resected non-small cell lung cancer. PLoS One. 2014;9(9):e106914.

35. Liu X, Sun X, Liu J, et al. Preoperative C-reactive protein/albumin ratio predicts prognosis of patients after curative resection for gastric cancer. Transl Oncol. 2015;8(4):339-45.

36. Saito H, Kono Y, Murakami Y, et al. Prognostic significance of the preoperative ratio of $\mathrm{C}$-reactive protein to albumin and neutrophil-lymphocyte ratio in gastric cancer patients. World J Surg. 2018;42(6):1819-25.

37. Shimada H, Takiguchi N, Kainuma O, et al. High preoperative neutrophil-lymphocyte ratio predicts poor survival in patients with gastric cancer. Gastric Cancer. 2010;13(3):170-6.

38. Sakuramoto S, Sasako M, Yamaguchi T, et al. Adjuvant chemotherapy for gastric cancer with S-1, an oral fluoropyrimidine. $\mathrm{N}$ Engl J Med. 2007;357(18):1810-20.
39. Sun P, Xiang JB, Chen ZY. Meta-analysis of adjuvant chemotherapy after radical surgery for advanced gastric cancer. Br J Surg. 2009;96(1):26-33.

40. Cheong J-H, Yang H-K, Kim H, et al. Predictive test for chemotherapy response in resectable gastric cancer: a multi-cohort, retrospective analysis. Lancet Oncol. 2018;19(5):629-38.

41. An JY, Kim H, Cheong JH, et al. Microsatellite instability in sporadic gastric cancer: its prognostic role and guidance for 5-FU based chemotherapy after R0 resection. Int $\mathrm{J}$ Cancer. 2012;131(2):505-11.

42. Choi YY, Kim H, Shin SJ, et al. Microsatellite instability and programmed cell death-ligand 1 expression in stage II/III gastric cancer: post hoc analysis of the CLASSIC randomized controlled study. Ann Surg. 2018. https://doi.org/10.1097/SLA.0000000000 002803 .

Publisher's Note Springer Nature remains neutral with regard to jurisdictional claims in published maps and institutional affiliations.

\section{Affiliations}

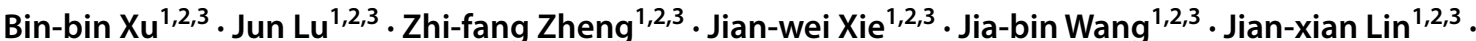 Qi-yue Chen ${ }^{1,2,3} \cdot$ Long-long Cao ${ }^{1,2,3} \cdot$ Mi Lin $^{1,2,3} \cdot$ Ru-hong Tu${ }^{1,2,3} \cdot$ Ze-ning Huang ${ }^{1,2,3} \cdot$ Ju-li Lin ${ }^{1,2,3}$. Chao-hui Zheng ${ }^{1,2,3}$. Chang-ming Huang ${ }^{1,2,3} \cdot$ Ping $\mathrm{Li}^{1,2,3}$}

Chao-hui Zheng wwkzch@163.com

$\triangle$ Chang-ming Huang hcmlr2002@163.com

Ping Li pingli811002@163.com
1 Department of Gastric Surgery, Fujian Medical University Union Hospital, No. 29 Xinquan Road, Fuzhou 350001, Fujian, China

2 Department of General Surgery, Fujian Medical University Union Hospital, Fuzhou, China

3 Key Laboratory of Ministry of Education of Gastrointestinal Cancer, Fujian Medical University, Fuzhou, Fujian, China 